\title{
Global stability of an epidemic model with stage structure and nonlinear incidence rates in a heterogeneous host population
}

\author{
Baodan Tian ${ }^{1,2 *}$, Yunguo Jin ${ }^{2,3}$, Shouming Zhong ${ }^{2}$ and Ning Chen ${ }^{1}$
}

\section{"Correspondence:}

tianbaodan@swust.edu.cn

'School of Science, Southwest

University of Science and

Technology, Mianyang, 621010 China

${ }^{2}$ School of Mathematical Sciences, University of Electronic Science and Technology of China, Chengdu, 611731, China

Full list of author information is available at the end of the article

\begin{abstract}
In this paper, we study an epidemic model with stage structure and latency spreading in a heterogeneous host population. We show that if the disease-free equilibrium exists, then the global dynamics are determined by the basic reproduction number $R_{0}$. We prove that the disease-free equilibrium is globally asymptotically stable when $R_{0} \leq 1$; and there exists a unique endemic equilibrium which is globally asymptotically stable when $R_{0}>1$. The global stability of the endemic equilibrium is also proved by using a graph-theoretic approach to the method of Lyapunov functions. Finally, numerical simulations are given to illustrate the main theoretical results.
\end{abstract}

Keywords: heterogeneous host; epidemic model; stage structure; nonlinear incidence rate; Lyapunov function

\section{Introduction}

A heterogeneous host population can be divided into several homogeneous groups according to models of transmission, contact patterns, or geographic distributions. Multigroup epidemic models have been proposed in the literature of mathematical epidemiology to describe the transmission dynamics of infectious diseases in heterogeneous host populations, such as measles, mumps, gonorrhea, HIV/AIDS, West-Nile virus and vectorborne diseases such as malaria. Various forms of multi-group epidemic models have subsequently been studied to understand the mechanism of disease transmission. One of the most important subjects in this field is to obtain a threshold that determines the persistence or extinction of a disease. Guo et al. in [1] developed a graph-theoretic approach to prove the global asymptotic stability of a unique endemic equilibrium of a multi-group epidemic model. By applying the idea, global stability of endemic equilibrium for several classes of multi-group epidemic models was investigated in [1-10].

In the real world, some epidemics, such as malaria, dengue, fever, gonorrhea and bacterial infections, may have a different ability to transmit the infections in different ages. For example, measles and varicella always occur in juveniles, while it is reasonable to consider the transmission of diseases such as typhus, diphtheria in adult population. In recent years, epidemic models with stage structure have been studied in many papers [11-17]. For some disease (for example, tuberculosis, influenza, measles), on adequate contact with an

(c) 2015 Tian et al. This article is distributed under the terms of the Creative Commons Attribution 4.0 International License (http://creativecommons.org/licenses/by/4.0/), which permits unrestricted use, distribution, and reproduction in any medium, provided you give appropriate credit to the original author(s) and the source, provide a link to the Creative Commons license, and indicate if changes were made. 
infective, a susceptible individual becomes exposed, that is, infected but not infective. This individual remains in the exposed class for a certain period before becoming infective (see, for example, [18-22]).

In this paper, we formulate an epidemic model with latency spreading in a heterogeneous host population. Let $S_{k}^{(1)}, S_{k}^{(2)}, E_{k}, I_{k}$ and $R_{k}$ denote the immature susceptible, mature susceptible, infected but non-infectious, infectious and recovered population in the $k$ th group, respectively. The disease incidence in the $k$ th group can be calculated as

$$
\sum_{i=1}^{2} S_{k}^{(i)} \sum_{j=1}^{n} \beta_{k j}^{(i)} G_{j}\left(I_{j}\right),
$$

where the sum takes into account cross-infections from all groups and $\beta_{k j}^{(i)}$ is the transmission coefficient between compartments $S_{k}^{(i)}$ and $I_{j} . G_{j}\left(I_{j}\right)$ includes some special incidence functions in the literature. For instance, $G_{j}\left(I_{j}\right)=\frac{I_{j}}{1+\alpha_{j} I_{j}}$ (saturation effect). Let $d_{k}^{(1)}$ and $d_{k}^{(2)}$ represent death rates of $S_{k}^{(1)}$ and $S_{k}^{(2)}$ populations, respectively. Then we obtain the following model for a disease with latency:

$$
\left\{\begin{array}{l}
\dot{S}_{k}^{(1)}=\varphi_{k}\left(S_{k}^{(1)}\right)-\sum_{j=1}^{n} \beta_{k j}^{(1)} S_{k}^{(1)} G_{j}\left(I_{j}\right)-a_{k} S_{k}^{(1)}, \\
\dot{S}_{k}^{(2)}=a_{k} S_{k}^{(1)}-\sum_{j=1}^{n} \beta_{k j}^{(2)} S_{k}^{(2)} G_{j}\left(I_{j}\right)-d_{k}^{(2)} S_{k}^{(2)}, \\
\dot{E}_{k}=\sum_{i=1}^{2} \sum_{j=1}^{n} \beta_{k j}^{(i)} S_{k}^{(i)} G_{j}\left(I_{j}\right)-\left(d_{k}+\eta_{k}\right) E_{k}, \\
\dot{I}_{k}=\eta_{k} E_{k}-\left(d_{k}+\mu_{k}+\gamma_{k}\right) I_{k}, \\
\dot{R}_{k}=\gamma_{k} I_{k}-d_{k} R_{k}, \quad k=1,2, \ldots, n,
\end{array}\right.
$$

where $\varphi_{k}\left(S_{k}^{(1)}\right)$ denotes the net growth rate of the immature susceptible class in the $k$ th group (a typical form of $\varphi_{k}\left(S_{k}^{(1)}\right)$ is $\varphi_{k}\left(S_{k}^{(1)}\right)=b_{k}-d_{k}^{(1)} S_{k}^{(1)}$ with $b_{k}$ the recruitment constant and $d_{k}^{(1)}$ the natural death rate). $a_{k}$ is the conversion rate from an immature individual to a mature individual in group $k . \eta_{k}$ represents the rate of becoming infectious after a latent period in the $k$ th group. $d_{k}, \mu_{k}$ and $\gamma_{k}$ are the natural death rate, the disease-related death rate and the recovery rate in the $k$ th group, respectively. All parameter values are assumed to be nonnegative and $a_{k}, \eta_{k}, d_{k}^{(i)}, d_{k}>0$.

Remark The model (1) can be regarded as an SVEIR model such that $S_{k}^{(1)}$ is unvaccinated and $S_{k}^{(2)}$ is vaccinated with vaccination rate $a_{k}$. References studied on the SVEIR model can be seen in $[23,24]$ and so on.

Since the variable $R_{k}$ does not appear in the remaining four equations of (1), if we denote $m_{k}:=d_{k}+\mu_{k}+\gamma_{k}$, then we can obtain the following reduced system:

$$
\left\{\begin{array}{l}
\dot{S}_{k}^{(1)}=\varphi_{k}\left(S_{k}^{(1)}\right)-\sum_{j=1}^{n} \beta_{k j}^{(1)} S_{k}^{(1)} G_{j}\left(I_{j}\right)-a_{k} S_{k}^{(1)}, \\
\dot{S}_{k}^{(2)}=a_{k} S_{k}^{(1)}-\sum_{j=1}^{n} \beta_{k j}^{(2)} S_{k}^{(2)} G_{j}\left(I_{j}\right)-d_{k}^{(2)} S_{k}^{(2)}, \\
\dot{E_{k}}=\sum_{i=1}^{2} \sum_{j=1}^{n} \beta_{k j}^{(i)} S_{k}^{(i)} G_{j}\left(I_{j}\right)-\left(d_{k}+\eta_{k}\right) E_{k}, \\
\dot{I_{k}}=\eta_{k} E_{k}-m_{k} I_{k}, \quad k=1,2, \ldots, n .
\end{array}\right.
$$

The initial conditions for system (2) are

$$
S_{k}^{(1)}(0)>0, \quad S_{k}^{(2)}(0)>0, \quad E_{k}(0)>0, \quad I_{k}(0)>0, \quad k=1,2, \ldots, n .
$$


The organization of this paper is as follows. In the next section, we prove some preliminary results for system (2). In Section 3, the main theorem of this paper is stated and proved. In the last section, a brief discussion and numerical simulations which support our theoretical analysis are given.

\section{Preliminaries}

We assume:

(A1) $\varphi_{k}$ and $G_{k}$ are Lipschitz on $[0,+\infty)$;

(A2) $\varphi_{k}$ is strictly decreasing on $[0,+\infty)$, and there exists $S_{k 0}^{(1)}>0$ such that

$$
\varphi_{k}\left(S_{k 0}^{(1)}\right)-a_{k} S_{k 0}^{(1)}=0
$$

(A3) $\frac{G_{k}(x)}{x}$ is nonincreasing on $(0,+\infty)$ and

$$
\delta_{k}=\lim _{x \rightarrow 0} \frac{G_{k}(x)}{x}>0 \text { exists, } \quad k=1,2, \ldots, n .
$$

From our assumptions, it is clear that system (2) has a unique solution for any given initial conditions (3) and the solution remains nonnegative. If (A2) holds, then we see that system (2) has a disease-free equilibrium

$$
P_{0}=\left(S_{10}^{(1)}, S_{10}^{(2)}, \ldots, S_{n 0}^{(1)}, S_{n 0}^{(2)}, 0,0, \ldots, 0\right),
$$

where

$$
\varphi_{k}\left(S_{k 0}^{(1)}\right)=d_{k}^{(2)} S_{k 0}^{(2)}, \quad a_{k} S_{k 0}^{(1)}=d_{k}^{(2)} S_{k 0}^{(2)}, \quad k=1,2, \ldots, n .
$$

For two nonnegative $n$-square matrices $\mathbf{A}=\left(a_{k j}\right)$ and $\mathbf{B}=\left(a_{k j}\right)$, we write $\mathbf{A} \leq \mathbf{B}$ if $a_{k j} \leq b_{k j}$ for all $k$ and $j$, and $\mathbf{A}<\mathbf{B}$ if $\mathbf{A} \leq \mathbf{B}$ and $\mathbf{A} \neq \mathbf{B}$. Following [25], we set matrices

$$
\mathbf{F}:=\left(\sum_{i=1}^{2} \beta_{k j}^{(i)} S_{k 0}^{(i)} \delta_{j}\right)_{n \times n}, \quad \mathbf{V}:=\operatorname{diag}\left(\frac{m_{1}\left(d_{1}+\eta_{1}\right)}{\eta_{1}}, \frac{m_{2}\left(d_{2}+\eta_{2}\right)}{\eta_{2}}, \ldots, \frac{m_{n}\left(d_{n}+\eta_{n}\right)}{\eta_{n}}\right) .
$$

The next generation matrix for system (2) is

$$
\begin{aligned}
\mathbf{Q} & :=\mathbf{F V}^{-\mathbf{1}}=\left(\frac{\eta_{k} \sum_{i=1}^{2} \beta_{k j}^{(i)} S_{k 0}^{(i)} \delta_{j}}{m_{k}\left(d_{k}+\eta_{k}\right)}\right)_{n \times n} \\
& =\left[\begin{array}{ccc}
\frac{\eta_{1} \sum_{i=1}^{2} \beta_{11}^{(i)} S_{10}^{(i)} \delta_{1}}{m_{1}\left(d_{1}+\eta_{1}\right)} & \ldots & \frac{\eta_{1} \sum_{i=1}^{2} \beta_{1 n}^{(i)} S_{10}^{(i)} \delta_{n}}{m_{1}\left(d_{1}+\eta_{1}\right)} \\
\vdots & \ddots & \vdots \\
\frac{\eta_{n} \sum_{i=1}^{2} \beta_{n 1}^{(i)} S_{n 0}^{(i)} \delta_{1}}{m_{n}\left(d_{n}+\eta_{n}\right)} & \ldots & \frac{\eta_{n} \sum_{i=1}^{2} \beta_{n n}^{(i)} S_{n 0}^{(i)} \delta_{n}}{m_{n}\left(d_{n}+\eta_{n}\right)}
\end{array}\right] .
\end{aligned}
$$

Thus, we obtain the basic reproduction number $R_{0}$ for system (2) as

$$
R_{0}=\rho(\mathbf{Q}),
$$

where $\rho$ denotes the spectral radius. 
Let $N_{k}=S_{k}^{(1)}+S_{k}^{(2)}+E_{k}+I_{k}, \underline{d}_{k}=\min \left\{d_{k}^{(1)}, d_{k}^{(2)}, d_{k}, m_{k}\right\}, k=1,2, \ldots, n$. Then from (2) we have

$$
\dot{N}_{k} \leq \varphi_{k}\left(S_{k}^{(1)}\right)+d_{k}^{(1)} S_{k}^{(1)}-\underline{d}_{k} N_{k}
$$

We derive from (5) that the region

$$
\begin{aligned}
\Gamma= & \left\{\left(S_{1}^{(1)}, S_{1}^{(2)}, \ldots, S_{n}^{(1)}, S_{n}^{(2)}, E_{1}, \ldots, E_{n}, I_{1}, \ldots, I_{n}\right) \in \mathbb{R}_{+}^{4 n}: S_{k}^{(1)} \leq S_{k 0}^{(1)},\right. \\
& \left.S_{k}^{(2)} \leq S_{k 0}^{(2)}, S_{k}^{(1)}+S_{k}^{(2)}+E_{k}+I_{k} \leq \frac{\varphi_{k}(0)+d_{k}^{(1)} S_{k 0}^{(1)}}{\underline{d}_{k}}, k=1,2, \ldots, n\right\}
\end{aligned}
$$

is positively invariant with respect to (2). Let $\Gamma^{\circ}$ denote the interior of $\Gamma$.

\section{Main results}

In the section, we study the global stability of equilibria of system (2).

Theorem 3.1 Assume that (A1)-(A3) hold and $\mathbf{B}=\left(\sum_{i=1}^{2} \beta_{k j}^{(i)}\right)$ is irreducible.

(1) If $R_{0} \leq 1$, then $P_{0}$ is globally asymptotically stable in $\Gamma$;

(2) If $R_{0}>1$, then $P_{0}$ is unstable and system (2) admits at least one endemic equilibrium in $\Gamma^{\circ}$.

Proof Let

$$
\begin{aligned}
& \mathbf{S}=\left(S_{1}^{(1)}, S_{1}^{(2)}, \ldots, S_{n}^{(1)}, S_{n}^{(2)}\right), \quad \mathbf{S}^{\mathbf{0}}=\left(S_{10}^{(1)}, S_{10}^{(2)}, \ldots, S_{n 0}^{(1)}, S_{n 0}^{(2)}\right), \\
& \mathbf{I}=\left(I_{1}, I_{2}, \ldots, I_{n}\right), \quad \mathbf{Q}(\mathbf{S}, \mathbf{I})=\left(\frac{\sum_{i=1}^{2} \sum_{j=1}^{n} \eta_{k} \beta_{k j}^{(i)} S_{k}^{(i)} G_{j}\left(I_{j}\right)}{m_{k}\left(d_{k}+\eta_{k}\right) I_{j}}\right)_{n \times n} .
\end{aligned}
$$

Notice that $\mathbf{B}$ is irreducible, then $\mathbf{Q}(\mathbf{S}, \mathbf{I})$ and $\mathbf{Q}$ are irreducible. By (A3), we have $\mathbf{0} \leq$ $\mathbf{Q}(\mathbf{S}, \mathbf{I}) \leq \mathbf{Q}$. Hence $\mathbf{Q}(\mathbf{S}, \mathbf{I})+\mathbf{Q}$ is also irreducible. That is, $0 \leq \mathbf{Q}(\mathbf{S}, \mathbf{I})<\mathbf{Q}$ and $\mathbf{Q}(\mathbf{S}, \mathbf{I})+\mathbf{Q}$ is irreducible provided that $\mathbf{S} \neq \mathbf{S}^{\mathbf{0}}$. Thus, by [26], Corollary 1.5, p.27, $\rho(\mathbf{Q}(\mathbf{S}, \mathbf{I}))<\rho(\mathbf{Q})$ if $\mathbf{S} \neq \mathbf{S}^{\mathbf{0}}$. Since $\mathbf{Q}$ is irreducible, there exist $\omega_{k}>0, k=1,2, \ldots, n$, such that

$$
\left(\omega_{1}, \omega_{2}, \ldots, \omega_{n}\right) \rho(\mathbf{Q})=\left(\omega_{1}, \omega_{2}, \ldots, \omega_{n}\right) \mathbf{Q}
$$

Consider a Lyapunov functional

$$
L=\sum_{k=1}^{n} \frac{\omega_{k} \eta_{k}}{m_{k}\left(d_{k}+\eta_{k}\right)}\left[E_{k}+\frac{d_{k}+\eta_{k}}{\eta_{k}} I_{k}\right] .
$$

Differentiating $L$ along the solution of system (2), we obtain

$$
\begin{aligned}
\dot{L} & =\sum_{k=1}^{n} \frac{\omega_{k} \eta_{k}}{m_{k}\left(d_{k}+\eta_{k}\right)}\left[\sum_{i=1}^{2} \sum_{j=1}^{n} \beta_{k j}^{(i)} S_{k}^{(i)} G_{j}\left(I_{j}\right)-\frac{m_{k}\left(d_{k}+\eta_{k}\right)}{\eta_{k}} I_{k}\right] \\
& =\sum_{k=1}^{n} \omega_{k}\left[\frac{\sum_{i=1}^{2} \sum_{j=1}^{n} \eta_{k} \beta_{k j}^{(i)} S_{k}^{(i)} G_{j}\left(I_{j}\right)}{m_{k}\left(d_{k}+\eta_{k}\right)}-I_{k}\right]
\end{aligned}
$$




$$
\begin{aligned}
& =\left(\omega_{1}, \omega_{2}, \ldots, \omega_{n}\right)\left(\mathbf{Q}(\mathbf{S}, \mathbf{I}) \mathbf{I}^{T}-\mathbf{I}^{T}\right) \\
& \leq\left(\omega_{1}, \omega_{2}, \ldots, \omega_{n}\right)\left(\mathbf{Q} \mathbf{I}^{T}-\mathbf{I}^{T}\right) \\
& =[\rho(\mathbf{Q})-1]\left(\omega_{1}, \omega_{2}, \ldots, \omega_{n}\right) \mathbf{I}^{T} \leq 0 .
\end{aligned}
$$

If $R_{0}<1$, then $\dot{L}=0$ if and only if $\mathbf{I}^{T}=\mathbf{0}$. If $R_{0}=1$, then $\dot{L}=0$ implies

$$
\left(\omega_{1}, \omega_{2}, \ldots, \omega_{n}\right)\left(\mathbf{Q}(\mathbf{S}, \mathbf{I}) \mathbf{I}^{T}-\mathbf{I}^{T}\right)=0
$$

Therefore, $\dot{L}=0$ if and only if $\mathbf{I}=\mathbf{0}$, or $\mathbf{S}=\mathbf{S}^{\mathbf{0}}$. On the other hand, from the last equation in system (2), we see that $\mathbf{I}=\mathbf{0}$ implies that $E_{k}=0$ for $k=1,2, \ldots, n$. Hence, the largest invariant subset of the set, where $\dot{L}=0$, is the singleton $\left\{P_{0}\right\}$. By LaSalle's invariance principle, $P_{0}$ is globally asymptotically stable for $R_{0} \leq 1$.

If $R_{0}>1$ and $\mathbf{I} \neq \mathbf{0}$, then

$$
[\rho(\mathbf{Q})-1]\left(\omega_{1}, \omega_{2}, \ldots, \omega_{n}\right) \mathbf{I}^{T}>0
$$

Thus, by continuity, we have $\dot{L}=\left(\omega_{1}, \omega_{2}, \ldots, \omega_{n}\right)\left(\mathbf{Q}(\mathbf{S}, \mathbf{I}) \mathbf{I}^{T}-\mathbf{I}^{T}\right)>0$ in a neighborhood of $P_{0}$ in $\Gamma^{\circ}$. This implies that $P_{0}$ is unstable. From a uniform persistence result of [27] and a similar argument as in the proof of Proposition 3.3 of [28], we can deduce that the instability of $P_{0}$ implies the uniform persistence of system (2) in $\Gamma^{\circ}$. This together with the uniform boundedness of solutions of system (2) in $\Gamma^{\circ}$ implies that system (2) has an endemic equilibrium in $\Gamma^{\circ}$ (see Theorem 2.8.6 of [29] or Theorem D.3 of [30]). The proof is completed.

By Theorem 3.1, we have that if $\mathbf{B}=\left(\sum_{i=1}^{2} \beta_{k j}^{(i)}\right)$ is irreducible, (A1)-(A3) hold and $R_{0}>1$, then system (2) has an endemic equilibrium $P^{*}$ in $\Gamma^{\circ}$. Let

$$
P^{*}=\left(S_{1}^{(1) *}, S_{1}^{(2) *}, \ldots, S_{n}^{(1) *}, S_{n}^{(2) *}, E_{1}^{*}, \ldots, E_{n}^{*}, I_{1}^{*}, \ldots, I_{n}^{*}\right),
$$

then the components of $P^{*}$ satisfy

$$
\begin{aligned}
& \varphi_{k}\left(S_{k}^{(1) *}\right)=\sum_{i=1}^{2} S_{k}^{(i) *} \sum_{j=1}^{n} \beta_{k j}^{(i)} G_{j}\left(I_{j}^{*}\right)+d_{k}^{(2)} S_{k}^{(2) *}, \\
& a_{k}\left(S_{k}^{(1) *}\right)=S_{k}^{(2) *} \sum_{j=1}^{n} \beta_{k j}^{(2)} G_{j}\left(I_{j}^{*}\right)+d_{k}^{(2)} S_{k}^{(2) *}, \\
& \sum_{i=1}^{2} S_{k}^{(i) *} \sum_{j=1}^{n} \beta_{k j}^{(i)} G_{j}\left(I_{j}^{*}\right)=\left(d_{k}+\eta_{k}\right) E_{k}^{*}=\frac{m_{k}\left(d_{k}+\eta_{k}\right)}{\eta_{k}} I_{k}^{*}, \quad k=1,2, \ldots, n .
\end{aligned}
$$

Since $\varphi_{k}$ is strictly decreasing on $[0,+\infty)$, we have

$$
\left[\varphi_{k}\left(S_{k}^{(1)}\right)-\varphi_{k}\left(S_{k}^{(1) *}\right)\right]\left(1-\frac{S_{k}^{(1) *}}{S_{k}^{(1)}}\right) \leq 0,
$$

where equality holds if and only if $S_{k}^{(1)}=S_{k}^{(1) *}, k=1,2, \ldots, n$. 
We further make the following assumption:

(A4) $G_{k}$ is strictly increasing on $[0,+\infty)$, and

$$
\frac{G_{k}\left(x_{k}\right) I_{k}}{G_{k}\left(I_{k}\right) x_{k}}+\frac{G_{k}\left(I_{k}\right)}{G_{k}\left(x_{k}\right)}-\frac{I_{k}}{x_{k}}-1 \leq 0, \quad k=1,2, \ldots, n,
$$

where $x_{k}>0$ is chosen in an arbitrary way and equality holds if $I_{k}=x_{k}$.

Theorem 3.2 Assume that $\mathbf{B}=\left[\sum_{i=1}^{2} \beta_{k j}^{(i)}\right]$ is irreducible. If $R_{0}>1$, then $P^{*}$ is globally asymptotically stable.

Proof Set $\bar{\beta}_{k j}=\sum_{i=1}^{2} \beta_{k j}^{(i)} S_{k}^{(i) *} G_{j}\left(I_{j}^{*}\right), 1 \leq k, j \leq n$, and

$$
\bar{B}=\left[\begin{array}{cccc}
\sum_{l \neq 1} \bar{\beta}_{1 l} & -\bar{\beta}_{21} & \ldots & -\bar{\beta}_{n 1} \\
-\bar{\beta}_{12} & \sum_{l \neq 2} \bar{\beta}_{2 l} & \ldots & -\bar{\beta}_{n 1} \\
\vdots & \vdots & \ddots & \vdots \\
-\bar{\beta}_{1 n} & -\bar{\beta}_{2 n} & \ldots & \sum_{l \neq n} \bar{\beta}_{n l}
\end{array}\right]
$$

Then $\bar{B}$ is also irreducible. It follows from Lemma 2.1 of [1] that the solution space of the linear system

$$
\bar{B} \mathbf{v}=0
$$

has dimension 1 with a basis

$$
\mathbf{v}:=\left(v_{1}, v_{2}, \ldots, v_{n}\right)^{T}=\left(\xi_{1}, \xi_{2}, \ldots, \xi_{n}\right)^{T}
$$

where $\xi_{k}$ denotes the cofactor of the $k$ th diagonal entry of $\bar{B}$. Note that from (11) we have

$$
\sum_{j=1}^{n} \bar{B}_{k j} v_{k}=\sum_{j=1}^{n} \bar{B}_{j k} v_{j}, \quad k=1,2, \ldots, n
$$

From (13), we have

$$
\begin{aligned}
& \sum_{k=1}^{n} v_{k} \sum_{j=1}^{n} \sum_{i=1}^{2} \beta_{k j}^{(i)} S_{k}^{(i) *} G_{j}\left(I_{j}\right) \\
& =\sum_{k, j=1}^{n} \sum_{i=1}^{2} \beta_{j k}^{(i)} S_{j}^{(i) *} v_{j} G_{k}\left(I_{k}\right)=\sum_{k=1}^{n}\left[\sum_{j=1}^{n} \sum_{i=1}^{2} \beta_{j k}^{(i)} S_{j}^{(i) *} G_{k}\left(I_{k}^{*}\right) v_{j}\right] \frac{G_{k}\left(I_{k}\right)}{G_{k}\left(I_{k}^{*}\right)} \\
& =\sum_{k=1}^{n}\left[\sum_{j=1}^{n}\left(\bar{\beta}_{j k} v_{j}\right)\right] \frac{G_{k}\left(I_{k}\right)}{G_{k}\left(I_{k}^{*}\right)}=\sum_{k=1}^{n}\left[\sum_{j=1}^{n}\left(\bar{\beta}_{k j} v_{k}\right)\right] \frac{G_{k}\left(I_{k}\right)}{G_{k}\left(I_{k}^{*}\right)} \\
& =\sum_{k=1}^{n} v_{k} \sum_{j=1}^{n} \sum_{i=1}^{2} \beta_{k j}^{(i)} S_{k}^{(i) *} G_{j}\left(I_{j}^{*}\right) \frac{G_{k}\left(I_{k}\right)}{G_{k}\left(I_{k}^{*}\right)}
\end{aligned}
$$




\section{Consider a Lyapunov functional}

$$
\begin{aligned}
V= & \sum_{k=1}^{n} v_{k}\left[\sum_{i=1}^{2}\left(S_{k}^{(i)}-S_{k}^{(i) *}-S_{k}^{(i) *} \ln \frac{S_{k}^{(i)}}{S_{k}^{(i) *}}\right)+E_{k}-E_{k}^{*}\right. \\
& \left.-E_{k}^{*} \ln \frac{E_{k}}{E_{k}^{*}}+\frac{d_{k}+\eta_{k}}{\eta_{k}} \int_{I_{k}^{*}}^{I_{k}} \frac{G_{k}(x)-G_{k}\left(I_{k}^{*}\right)}{G_{k}(x)} d x\right] .
\end{aligned}
$$

Differentiating $V$ along the solution of system (2), we obtain

$$
\begin{aligned}
\dot{V}= & \sum_{k=1}^{n} v_{k}\left\{\varphi_{k}\left(S_{k}^{(1)}\right)-d_{k}^{(2)} S_{k}^{(2)}-\frac{m_{k}\left(d_{k}+\eta_{k}\right)}{\eta_{k}} I_{k}\right. \\
& -\frac{S_{k}^{(1) *}}{S_{k}^{(1)}}\left[\varphi_{k}\left(S_{k}^{(1)}\right)-S_{k}^{(1)} \sum_{j=1}^{n} \beta_{k j}^{(1)} G_{j}\left(I_{j}\right)-a_{k} S_{k}^{(1)}\right] \\
& -\frac{S_{k}^{(2) *}}{S_{k}^{(2)}}\left[a_{k} S_{k}^{(1)}-S_{k}^{(2)} \sum_{j=1}^{n} \beta_{k j}^{(2)} G_{j}\left(I_{j}\right)-d_{k}^{(2)} S_{k}^{(2)}\right] \\
& -\frac{E_{k}^{*}}{E_{k}}\left[\sum_{i=1}^{2} \sum_{j=1}^{n} \beta_{k j}^{(i)} S_{k}^{(i)} G_{j}\left(I_{j}\right)-\left(d_{k}+\eta_{k}\right) E_{k}\right] \\
& \left.-\frac{G_{k}\left(I_{k}^{*}\right)}{G_{k}\left(I_{k}\right)}\left[\left(d_{k}+\eta_{k}\right) E_{k}-\frac{m_{k}\left(d_{k}+\eta_{k}\right)}{\eta_{k}} I_{k}\right]\right\} \\
= & \sum_{k=1}^{n} v_{k}\left\{\varphi_{k}\left(S_{k}^{(1)}\right)\left(1-\frac{S_{k}^{(1) *}}{S_{k}^{(1)}}\right)+d_{k}^{(2)} S_{k}^{(2) *}\left(1-\frac{S_{k}^{(2)}}{S_{k}^{(2) *}}\right)\right. \\
& +\frac{m_{k}\left(d_{k}+\eta_{k}\right)}{\eta_{k} S_{k}^{(1) *}\left(1-\frac{S_{k}^{*}}{S_{k}^{(1) *} G_{k}\left(I_{k}^{*}\right)} I_{k}^{*} G_{k}\left(I_{k}\right)\right.}-\frac{m_{k}\left(d_{k}+\eta_{k}\right)}{\eta_{k}} I_{k}^{*} \frac{I_{k}}{I_{k}^{*}} S_{k}^{(i) *} \sum_{j=1}^{n} \beta_{k j}^{(i)} G_{j}\left(I_{j}\right) \\
& -\frac{E_{k}^{*}}{E_{k}} \sum_{i=1}^{2}-S_{k}^{(i)} \sum_{j=1}^{n} \beta_{k j}^{(i)} G_{j}\left(I_{j}\right)+\left(d_{k}+\eta_{k}\right) E_{k}^{*}\left(1-\frac{E_{k} G_{k}\left(I_{k}^{*}\right)}{E_{k}^{*} G_{k}\left(I_{k}\right)}\right) \\
& \\
& \\
& \\
&
\end{aligned}
$$

From (7) and (8), we have

$$
\begin{aligned}
& \dot{V}=\sum_{k=1}^{n} v_{k}\left\{\varphi_{k}\left(S_{k}^{(1)}\right)\left(1-\frac{S_{k}^{(1) *}}{S_{k}^{(1)}}\right)+d_{k}^{(2)} S_{k}^{(2) *}\left(1-\frac{S_{k}^{(2)}}{S_{k}^{(2) *}}\right)\right. \\
& -\frac{E_{k}^{*}}{E_{k}} \sum_{i=1}^{2} S_{k}^{(i)} \sum_{j=1}^{n} \beta_{k j}^{(i)} G_{j}\left(I_{j}\right) \\
& +\left[S_{k}^{(2) *} \sum_{j=1}^{n} \beta_{k j}^{(2)} G_{j}\left(I_{j}^{*}\right)+d_{k}^{(2)} S_{k}^{(2) *}\right]\left(1-\frac{S_{k}^{(1)} S_{k}^{(2) *}}{S_{k}^{(1) *} S_{k}^{(2)}}\right) \\
& \left.+\sum_{i=1}^{2} S_{k}^{(i) *} \sum_{j=1}^{n} \beta_{k j}^{(i)} G_{j}\left(I_{j}^{*}\right)\left[1-\frac{E_{k} G_{k}\left(I_{k}^{*}\right)}{E_{k}^{*} G_{k}\left(I_{k}\right)}+\frac{G_{j}\left(I_{j}\right)}{G_{j}\left(I_{j}^{*}\right)}+\frac{I_{k} G_{k}\left(I_{k}^{*}\right)}{I_{k}^{*} G_{k}\left(I_{k}\right)}-\frac{I_{k}}{I_{k}^{*}}\right]\right\} \text {. }
\end{aligned}
$$


By (10) and (14), we obtain

$$
\begin{aligned}
\dot{V} \leq & \sum_{k=1}^{n} v_{k}\left\{\varphi_{k}\left(S_{k}^{(1)}\right)\left(1-\frac{S_{k}^{(1) *}}{S_{k}^{(1)}}\right)+d_{k}^{(2)} S_{k}^{(2) *}\left(1-\frac{S_{k}^{(2)}}{S_{k}^{(2) *}}\right)\right. \\
& -\frac{E_{k}^{*}}{E_{k}} \sum_{i=1}^{2} S_{k}^{(i)} \sum_{j=1}^{n} \beta_{k j}^{(i)} G_{j}\left(I_{j}\right)+\left[S_{k}^{(2) *} \sum_{j=1}^{n} \beta_{k j}^{(2)} G_{j}\left(I_{j}^{*}\right)+d_{k}^{(2)} S_{k}^{(2) *}\right] \\
& \left.\times\left(1-\frac{S_{k}^{(1)} S_{k}^{(2) *}}{S_{k}^{(1) *} S_{k}^{(2)}}\right)+\sum_{i=1}^{2} S_{k}^{(i) *} \sum_{j=1}^{n} \beta_{k j}^{(i)} G_{j}\left(I_{j}^{*}\right)\left[2-\frac{E_{k} G_{k}\left(I_{k}^{*}\right)}{E_{k}^{*} G_{k}\left(I_{k}\right)}\right]\right\}=: B_{1} .
\end{aligned}
$$

From (6), we know that

$$
\varphi_{k}\left(S_{k}^{(1) *}\right)\left(1-\frac{S_{k}^{(1) *}}{S_{k}^{(1)}}\right)=\left(\sum_{i=1}^{2} S_{k}^{(i) *} \sum_{j=1}^{n} \beta_{k j}^{(i)} G_{j}\left(I_{j}^{*}\right)+d_{k}^{(2)} S_{k}^{(2) *}\right)\left(1-\frac{S_{k}^{(1) *}}{S_{k}^{(1)}}\right) .
$$

By (16), we can rewrite $B_{1}$ as

$$
\begin{aligned}
\dot{V}= & \sum_{k=1}^{n} v_{k}\left\{\left[\varphi_{k}\left(S_{k}^{(1)}\right)-\varphi_{k}\left(S_{k}^{(1) *}\right)\right]\left(1-\frac{S_{k}^{(1) *}}{S_{k}^{(1)}}\right)\right. \\
& +d_{k}^{(2)} S_{k}^{(2) *}\left(3-\frac{S_{k}^{(1) *}}{S_{k}^{(1)}}-\frac{S_{k}^{(1)} S_{k}^{(2) *}}{S_{k}^{(1) *} S_{k}^{(2)}}-\frac{S_{k}^{(2)}}{S_{k}^{(2) *}}\right) \\
& -\frac{E_{k}^{*}}{E_{k}} \sum_{i=1}^{2} S_{k}^{(i)} \sum_{j=1}^{n} \beta_{k j}^{(i)} G_{j}\left(I_{j}\right)+S_{k}^{(2) *} \sum_{j=1}^{n} \beta_{k j}^{(2)} G_{j}\left(I_{j}^{*}\right) \\
& \times\left(2-\frac{S_{k}^{(1) *}}{S_{k}^{(1)}}-\frac{S_{k}^{(1)} S_{k}^{(2) *}}{S_{k}^{(1) *} S_{k}^{(2)}}\right)+S_{k}^{(1) *} \sum_{j=1}^{n} \beta_{k j}^{(1)} G_{j}\left(I_{j}^{*}\right)\left(1-\frac{S_{k}^{(1) *}}{S_{k}^{(1)}}\right) \\
& \left.+\sum_{i=1}^{2} S_{k}^{(i) *} \sum_{j=1}^{n} \beta_{k j}^{(i)} G_{j}\left(I_{j}^{*}\right)\left[2-\frac{E_{k} G_{k}\left(I_{k}^{*}\right)}{E_{k}^{*} G_{k}\left(I_{k}\right)}\right]\right\} .
\end{aligned}
$$

By (9) and the arithmetic-geometric mean, we easily see that

$$
\begin{aligned}
B_{1} \leq & \sum_{k=1}^{n} v_{k}\left\{-\frac{E_{k}^{*}}{E_{k}} \sum_{i=1}^{2} S_{k}^{(i)} \sum_{j=1}^{n} \beta_{k j}^{(i)} G_{j}\left(I_{j}\right)\right. \\
& +S_{k}^{(2) *} \sum_{j=1}^{n} \beta_{k j}^{(2)} G_{j}\left(I_{j}^{*}\right)\left(2-\frac{S_{k}^{(1) *}}{S_{k}^{(1)}}-\frac{S_{k}^{(1)} S_{k}^{(2) *}}{S_{k}^{(1) *} S_{k}^{(2)}}\right) \\
& +S_{k}^{(1) *} \sum_{j=1}^{n} \beta_{k j}^{(1)} G_{j}\left(I_{j}^{*}\right)\left(1-\frac{S_{k}^{(1) *}}{S_{k}^{(1)}}\right) \\
& \left.+\sum_{i=1}^{2} S_{k}^{(i) *} \sum_{j=1}^{n} \beta_{k j}^{(i)} G_{j}\left(I_{j}^{*}\right)\left[2-\frac{E_{k} G_{k}\left(I_{k}^{*}\right)}{E_{k}^{*} G_{k}\left(I_{k}\right)}\right]\right\}=: B_{2} .
\end{aligned}
$$


We can rewrite $B_{2}$ as

$$
\begin{aligned}
B_{2}= & \sum_{k=1}^{n} v_{k}\left\{S _ { k } ^ { ( 2 ) * } \sum _ { j = 1 } ^ { n } \beta _ { k j } ^ { ( 2 ) } G _ { j } ( I _ { j } ^ { * } ) \left[3-\frac{S_{k}^{(1) *}}{S_{k}^{(1)}}-\frac{S_{k}^{(1)} S_{k}^{(2) *}}{S_{k}^{(1) *} S_{k}^{(2)}}\right.\right. \\
& \left.-\frac{S_{k}^{(2)} E_{k}^{*} G_{j}\left(I_{j}\right)}{S_{k}^{(2) *} E_{k} G_{j}\left(I_{j}^{*}\right)}\right]+S_{k}^{(1) *} \sum_{j=1}^{n} \beta_{k j}^{(1)} G_{j}\left(I_{j}^{*}\right)\left[2-\frac{S_{k}^{(1) *}}{S_{k}^{(1)}}\right. \\
& \left.\left.-\frac{S_{k}^{(1)} E_{k}^{*} G_{j}\left(I_{j}\right)}{S_{k}^{(1) *} E_{k} G_{j}\left(I_{j}^{*}\right)}\right]+\sum_{i=1}^{2} S_{k}^{(i) *} \sum_{j=1}^{n} \beta_{k j}^{(i)} G_{j}\left(I_{j}^{*}\right)\left[1-\frac{E_{k} G_{k}\left(I_{k}^{*}\right)}{E_{k}^{*} G_{k}\left(I_{k}\right)}\right]\right\} .
\end{aligned}
$$

By the arithmetic-geometric mean, we have that

$$
\begin{aligned}
B_{2} \leq & \sum_{k=1}^{n} v_{k}\left\{3 S_{k}^{(2) *} \sum_{j=1}^{n} \beta_{k j}^{(2)} G_{j}\left(I_{j}^{*}\right)\left[1-\left(\frac{E_{k}^{*} G_{j}\left(I_{j}\right)}{E_{k} G_{j}\left(I_{j}^{*}\right)}\right)^{\frac{1}{3}}\right]\right. \\
& +2 S_{k}^{(1) *} \sum_{j=1}^{n} \beta_{k j}^{(1)} G_{j}\left(I_{j}^{*}\right)\left[1-\left(\frac{E_{k}^{*} G_{j}\left(I_{j}\right)}{E_{k} G_{j}\left(I_{j}^{*}\right)}\right)^{\frac{1}{2}}\right] \\
& \left.+\sum_{i=1}^{2} S_{k}^{(i) *} \sum_{j=1}^{n} \beta_{k j}^{(i)} G_{j}\left(I_{j}^{*}\right)\left[1-\frac{E_{k} G_{k}\left(I_{k}^{*}\right)}{E_{k}^{*} G_{k}\left(I_{k}\right)}\right]\right\}=: B_{3} .
\end{aligned}
$$

We can rewrite $B_{3}$ as

$$
\begin{aligned}
B_{3}= & \sum_{k=1}^{n} v_{k}\left\{3 S_{k}^{(2) *} \sum_{j=1}^{n} \beta_{k j}^{(2)} G_{j}\left(I_{j}^{*}\right)\left[1-\left[\frac{E_{k}^{*} G_{j}\left(I_{j}\right)}{E_{k} G_{j}\left(I_{j}^{*}\right)}\right]^{\frac{1}{3}}+\ln \left[\frac{E_{k}^{*} G_{j}\left(I_{j}\right)}{E_{k} G_{j}\left(I_{j}^{*}\right)}\right]^{\frac{1}{3}}\right]\right. \\
& +2 S_{k}^{(1) *} \sum_{j=1}^{n} \beta_{k j}^{(1)} G_{j}\left(I_{j}^{*}\right)\left[1-\left[\frac{E_{k}^{*} G_{j}\left(I_{j}\right)}{E_{k} G_{j}\left(I_{j}^{*}\right)}\right]^{\frac{1}{2}}+\ln \left[\frac{E_{k}^{*} G_{j}\left(I_{j}\right)}{E_{k} G_{j}\left(I_{j}^{*}\right)}\right]^{\frac{1}{2}}\right] \\
& -\sum_{i=1}^{2} S_{k}^{(i) *} \sum_{j=1}^{n} \beta_{k j}^{(i)} G_{j}\left(I_{j}^{*}\right) \ln \frac{E_{k}^{*} G_{j}\left(I_{j}\right)}{E_{k} G_{j}\left(I_{j}^{*}\right)} \\
& +\sum_{i=1}^{2} S_{k}^{(i) *} \sum_{j=1}^{n} \beta_{k j}^{(i)} G_{j}\left(I_{j}^{*}\right)\left[1-\frac{E_{k} G_{k}\left(I_{k}^{*}\right)}{E_{k}^{*} G_{k}\left(I_{k}\right)}+\ln \frac{E_{k} G_{k}\left(I_{k}^{*}\right)}{E_{k}^{*} G_{k}\left(I_{k}\right)}\right] \\
& \left.-\sum_{i=1}^{2} S_{k}^{(i) *} \sum_{j=1}^{n} \beta_{k j}^{(i)} G_{j}\left(I_{j}^{*}\right) \ln \frac{E_{k} G_{k}\left(I_{k}^{*}\right)}{E_{k}^{*} G_{k}\left(I_{k}\right)}\right\} .
\end{aligned}
$$

Using the fact that $1-x+\ln x \leq 0$, where equality holds if and only if $x=1$, we obtain

$$
\begin{aligned}
B_{3} & \leq \sum_{k=1}^{n} v_{k} \sum_{i=1}^{2} S_{k}^{(i) *} \sum_{j=1}^{n} \beta_{k j}^{(i)} G_{j}\left(I_{j}^{*}\right)\left[-\ln \frac{E_{k}^{*} G_{j}\left(I_{j}\right)}{E_{k} G_{j}\left(I_{j}^{*}\right)}-\ln \frac{E_{k} G_{k}\left(I_{k}^{*}\right)}{E_{k}^{*} G_{k}\left(I_{k}\right)}\right] \\
& =\sum_{k=1}^{n} v_{k} \sum_{i=1}^{2} S_{k}^{(i) *} \sum_{j=1}^{n} \beta_{k j}^{(i)} G_{j}\left(I_{j}^{*}\right) \ln \frac{G_{k}\left(I_{k}\right) G_{j}\left(I_{j}^{*}\right)}{G_{k}\left(I_{k}^{*}\right) G_{j}\left(I_{j}\right)} \\
& =\sum_{k=1}^{n} v_{k} \sum_{j=1}^{n} \bar{\beta}_{k j} \ln \frac{G_{k}\left(I_{k}\right) G_{j}\left(I_{j}^{*}\right)}{G_{k}\left(I_{k}^{*}\right) G_{j}\left(I_{j}\right)} .
\end{aligned}
$$


In the following, we will show that

$$
H_{n}:=\sum_{k=1}^{n} v_{k} \sum_{j=1}^{n} \bar{\beta}_{k j} \ln \frac{G_{k}\left(I_{k}\right) G_{j}\left(I_{j}^{*}\right)}{G_{k}\left(I_{k}^{*}\right) G_{j}\left(I_{j}\right)} \equiv 0
$$

We first give the proof of (20) for $n=2$, which would give a reader the basic yet clear ideas without being hidden by the complexity of terms caused by larger values of $n$. When $n=2$, we have

$$
H_{2}=\sum_{k=1}^{2} v_{k} \sum_{j=1}^{2} \bar{\beta}_{k j} \ln \frac{G_{k}\left(I_{k}\right) G_{j}\left(I_{j}^{*}\right)}{G_{k}\left(I_{k}^{*}\right) G_{j}\left(I_{j}\right)}
$$

Formula (12) gives $v_{1}=\bar{\beta}_{21}$ and $v_{2}=\bar{\beta}_{12}$ in this case. Expanding $H_{2}$ yields

$$
\begin{aligned}
H_{2}= & \bar{\beta}_{21} \bar{\beta}_{11} \ln \frac{G_{1}\left(I_{1}\right) G_{1}\left(I_{1}^{*}\right)}{G_{1}\left(I_{1}^{*}\right) G_{1}\left(I_{1}\right)}+\bar{\beta}_{12} \bar{\beta}_{22} \ln \frac{G_{2}\left(I_{2}\right) G_{2}\left(I_{2}^{*}\right)}{G_{2}\left(I_{2}^{*}\right) G_{2}\left(I_{2}\right)} \\
& +\bar{\beta}_{21} \bar{\beta}_{12} \ln \frac{G_{1}\left(I_{1}\right) G_{2}\left(I_{2}^{*}\right)}{G_{1}\left(I_{1}^{*}\right) G_{2}\left(I_{2}\right)}+\bar{\beta}_{12} \bar{\beta}_{21} \ln \frac{G_{2}\left(I_{2}\right) G_{1}\left(I_{1}^{*}\right)}{G_{2}\left(I_{2}^{*}\right) G_{1}\left(I_{1}\right)} \\
= & \bar{\beta}_{12} \bar{\beta}_{21}\left[\ln \frac{G_{1}\left(I_{1}\right) G_{2}\left(I_{2}^{*}\right)}{G_{1}\left(I_{1}^{*}\right) G_{2}\left(I_{2}\right)}+\ln \frac{G_{2}\left(I_{2}\right) G_{1}\left(I_{1}^{*}\right)}{G_{2}\left(I_{2}^{*}\right) G_{1}\left(I_{1}\right)}\right]=0 .
\end{aligned}
$$

For more general $n$, by a similar argument as in the proof of $\sum_{k, j=1}^{n} v_{k} \bar{\beta}_{k j} \ln \frac{E_{k}^{*} E_{j}}{E_{k} E_{j}^{*}} \equiv 0$ in [7], we obtain that

$$
\sum_{k=1}^{n} v_{k} \sum_{j=1}^{n} \bar{\beta}_{k j} \ln \frac{G_{k}\left(I_{k}\right) G_{j}\left(I_{j}^{*}\right)}{G_{k}\left(I_{k}^{*}\right) G_{j}\left(I_{j}\right)}=-\sum_{k, j=1}^{n} v_{k} \bar{\beta}_{k j} \ln \frac{G_{k}\left(I_{k}^{*}\right) G_{j}\left(I_{j}\right)}{G_{k}\left(I_{k}\right) G_{j}\left(I_{j}^{*}\right)} \equiv 0 .
$$

From (17)-(19), we see that if $\dot{V}=0$, then

$$
S_{k}^{(i)}=S_{k}^{(i) *}, \quad i=1,2, k=1,2, \ldots, n
$$

If (21) holds, it follows from (2) that

$$
\left\{\begin{array}{l}
0=\varphi_{k}\left(S_{k}^{(1) *}\right)-\sum_{j=1}^{n} \beta_{k j}^{(1)} S_{k}^{(1) *} G_{j}\left(I_{j}\right)-a_{k} S_{k}^{(1) *} \\
0=a_{k} S_{k}^{(1) *}-\sum_{j=1}^{n} \beta_{k j}^{(2)} S_{k}^{(2) *} G_{j}\left(I_{j}\right)-d_{k}^{(2)} S_{k}^{(2) *}
\end{array}\right.
$$

Then we obtain that

$$
\dot{E}_{k}=\left(\varphi_{k}\left(S_{k}^{(1) *}\right)-a_{k} S_{k}^{(1) *}\right)+\left(a_{k} S_{k}^{(1) *}-d_{k}^{(2)} S_{k}^{(2) *}\right)-\left(d_{k}+\eta_{k}\right) E_{k}
$$

This implies that

$$
\lim _{t \rightarrow+\infty} E_{k}=\frac{\left(\varphi_{k}\left(S_{k}^{(1) *}\right)-a_{k} S_{k}^{(1) *}\right)+\left(a_{k} S_{k}^{(1) *}-d_{k}^{(2)} S_{k}^{(2) *}\right)}{\left(d_{k}+\eta_{k}\right)}=E_{k}^{*} .
$$

By (22) and the fourth equation of system (2), we have

$$
\lim _{t \rightarrow+\infty} I_{k}=\frac{\eta_{k} E_{k}^{*}}{m_{k}}=I_{k}^{*}
$$


From (21)-(23) and the characteristics of $V$, we obtain that the largest invariant subset of the set, where $\dot{V}=0$, is the singleton $\left\{P^{*}\right\}$. By LaSalle's invariance principle, $P^{*}$ is globally asymptotically stable for $R_{0}>1$.

\section{Numerical examples}

For certain sexually transmitted diseases, AIDS/HIV for example, it is natural to consider two groups of people: a group of males and a group of females. Further, it is always assumed that there are two important age stages for the susceptible, a group of immature susceptible $S_{k}^{(1)}$ who are less than 18 years old, and a group of mature susceptible $S_{k}^{(2)}$ who are more than 18 years old. Thus, we consider the following model:

$$
\left\{\begin{array}{l}
\dot{S}_{k}^{(1)}=\varphi_{k}\left(S_{k}^{(1)}\right)-\sum_{j=1}^{2} \beta_{k j}^{(1)} S_{k}^{(1)} G_{j}\left(I_{j}\right)-a_{k} S_{k}^{(1)}, \\
\dot{S}_{k}^{(2)}=a_{k} S_{k}^{(1)}-\sum_{j=1}^{2} \beta_{k j}^{(2)} S_{k}^{(2)} G_{j}\left(I_{j}\right)-d_{k}^{(2)} S_{k}^{(2)}, \\
\dot{E_{k}}=\sum_{i=1}^{2} \sum_{j=1}^{2} \beta_{k j}^{(i)} S_{k}^{(i)} G_{j}\left(I_{j}\right)-\left(d_{k}+\eta_{k}\right) E_{k}, \\
\dot{I}_{k}=\eta_{k} E_{k}-m_{k} I_{k}, \quad k=1,2,
\end{array}\right.
$$

where $\varphi_{k}\left(S_{k}^{(1)}\right)=b_{k}-d_{k}^{(1)} S_{k}^{(1)}, G_{j}\left(I_{j}\right)=\frac{I_{j}}{1+\alpha_{j} I_{j}}$.

Clearly, (A1)-(A4) hold. We fix the parameters as follows:

$$
\begin{aligned}
& b_{1}=50, \quad b_{2}=30, \quad d_{1}^{(1)}=0.001, \quad d_{1}^{(2)}=0.2, \quad d_{2}^{(1)}=0.002, \\
& d_{2}^{(2)}=0.3, \quad d_{1}=0.1, \quad d_{2}=0.2, \quad \eta_{1}=0.1, \quad \eta_{2}=0.2, \\
& m_{1}=0.5, \quad m_{2}=0.6, \quad a_{1}=0.6, \quad a_{2}=0.5, \quad \alpha_{1}=\alpha_{2}=0.1 \text {. }
\end{aligned}
$$

Then we have $P_{0} \approx(83.1947,249.5840,59.7610,99.6016,0,0,0,0)$.

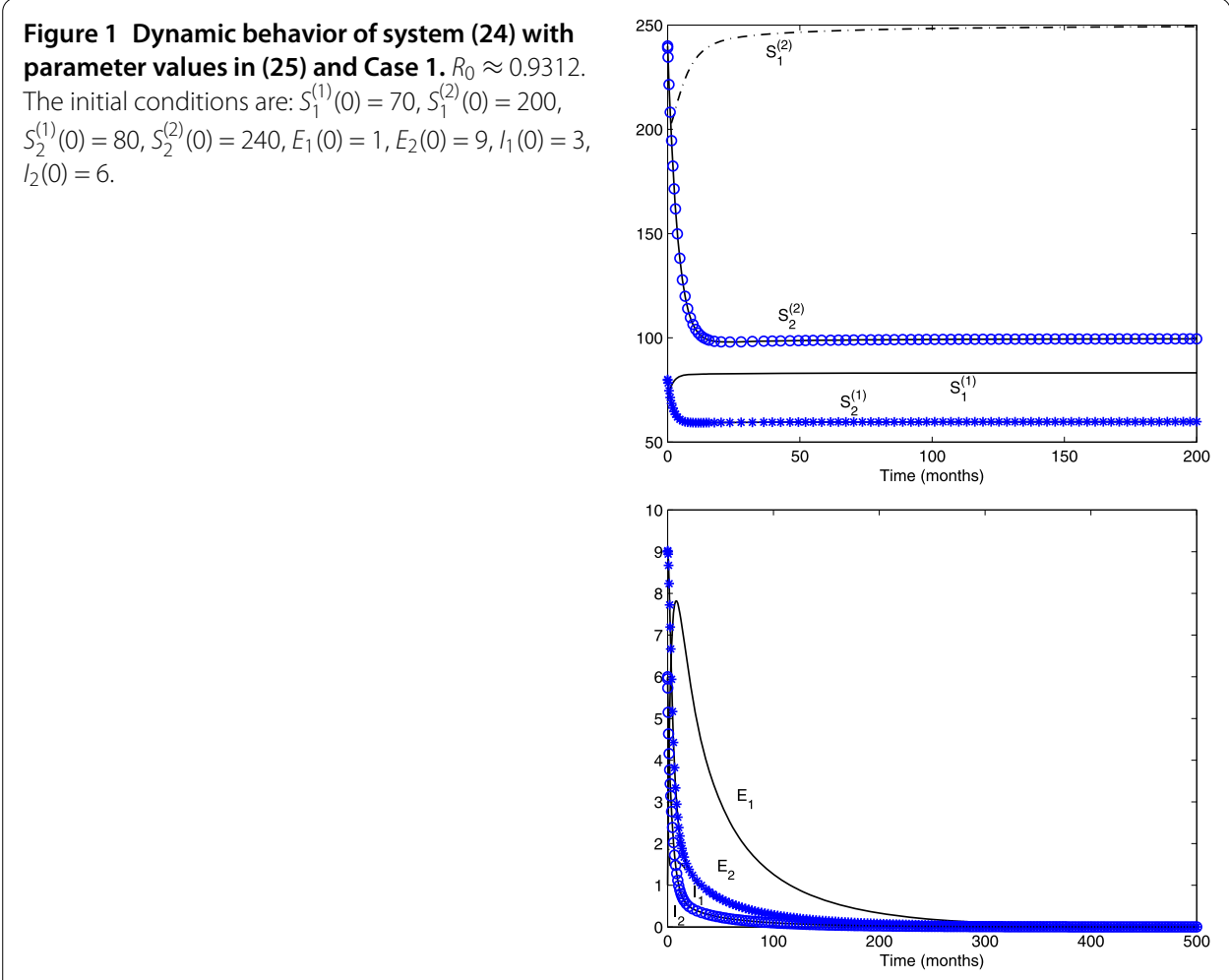




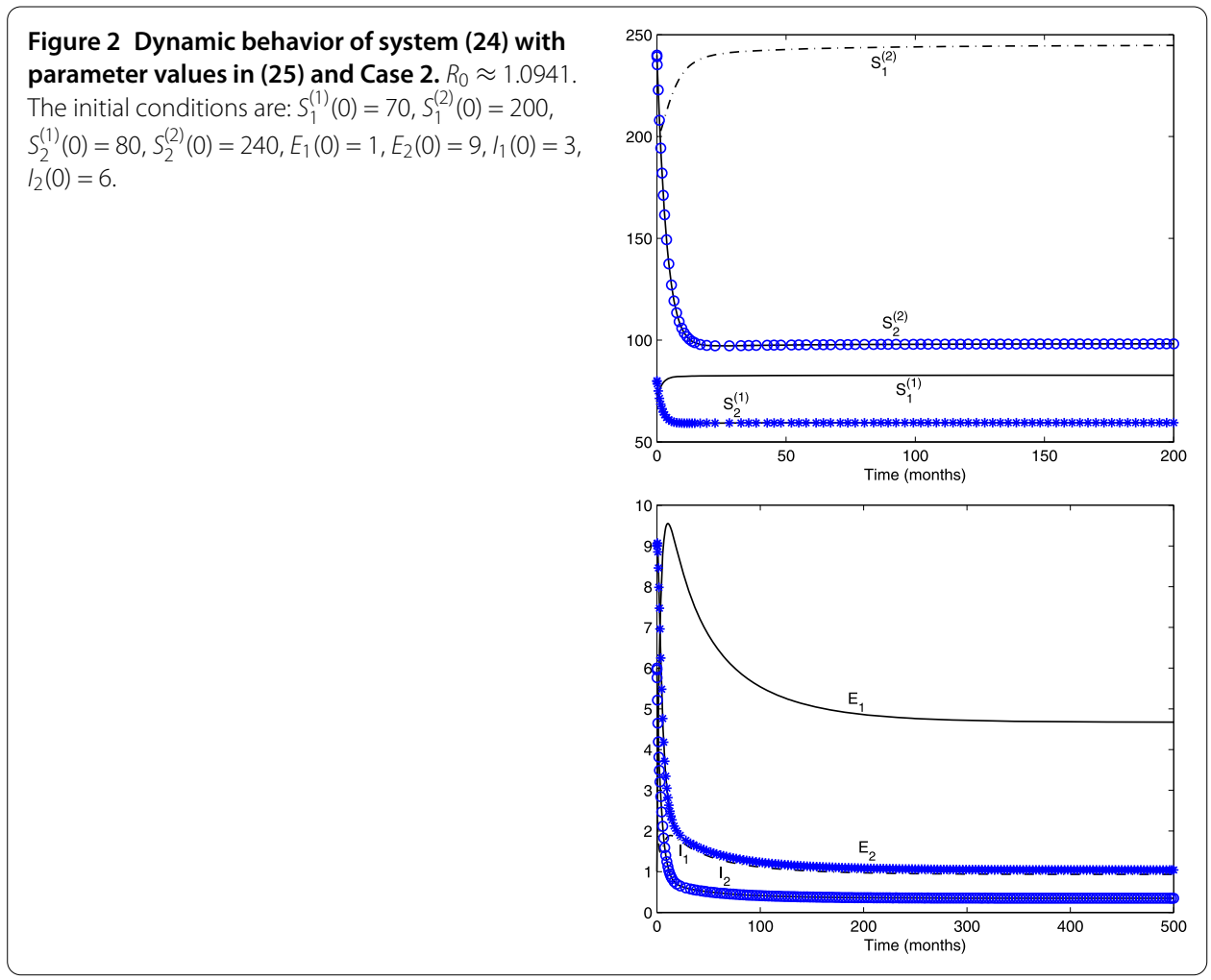

Case 1. If $\beta_{1 j}^{(1)}=\beta_{k 1}^{(2)}=0.002, \beta_{2 j}^{(1)}=\beta_{k 2}^{(2)}=0.002, k=1,2, j=1,2$, then we obtain

$$
\mathbf{Q} \approx\left(\begin{array}{cc}
0.6656 & 0.5546 \\
0.3187 & 0.2656
\end{array}\right), \quad R_{0} \approx 0.9312
$$

By Theorem 3.1, the disease dies out in both groups. Numerical simulation illustrates this fact (see Figure 1).

Case 2. If $\beta_{1 j}^{(1)}=\beta_{k 1}^{(2)}=0.0025, \beta_{2 j}^{(1)}=\beta_{k 2}^{(2)}=0.002, k=1,2, j=1,2$, then we have $P^{*} \approx$ $(82.7845,244.9127,59.4787,98.2113,4.6734,1.0441,0.9347,0.3480)$ and $R_{0} \approx 1.0941$.

By Theorem 3.2, the disease persists in both groups. Numerical simulation illustrates this fact (see Figure 2).

Competing interests

The authors declare that they have no competing interests.

\section{Authors' contributions}

All authors contributed equally and significantly in writing this paper. All authors read and approved the final paper.

\section{Author details}

${ }^{1}$ School of Science, Southwest University of Science and Technology, Mianyang, 621010, China. ${ }^{2}$ School of Mathematical Sciences, University of Electronic Science and Technology of China, Chengdu, 611731, China. ${ }^{3}$ School of Statistics, Chengdu University of Information Technology, Chengdu, 610103, China.

\section{Acknowledgements}

This work is supported by the National Natural Science Foundation of China (51349011), Scientific Research Fund of Sichuan Provincial Education Department (11ZB192, 14ZB0115) and Doctorial Research Fund of Southwest University of Science and Technology. 


\section{References}

1. Guo, H, Li, MY, Shuai, Z: Global stability of the endemic equilibrium of multigroup SIR epidemic models. Can. Appl. Math. Q. 14, 259-284 (2006)

2. Sun, R, Shi, J: Global stability of multigroup endemic model with group mixing and nonlinear incidence rates. Appl. Math. Comput. 218, 280-286 (2011)

3. Yuan, Z, Wang, L: Global stability of epidemiological models with group mixing and nonlinear incidence rates. Nonlinear Anal., Real World Appl. 11, 995-1004 (2011)

4. Kuniya, T: Global stability analysis with a discretization approach for an age-structured multigroup SIR epidemic model. Nonlinear Anal., Real World Appl. 12, 2640-2655 (2011)

5. Yuan, Z, Zou, X: Global threshold property in an epidemic model for disease with latency spreading in a heterogeneous host population. Nonlinear Anal., Real World Appl. 11, 3479-3490 (2011)

6. Sun, R: Global stability of the endemic equilibrium of multigroup SIR epidemic models with nonlinear incidence rates. Comput. Math. Appl. 60, 2286-2291 (2010)

7. Li, MY, Shuai, Z, Wang, C: Global stability of multi-group epidemic models with distributed delays. J. Math. Anal. Appl. 361, 38-47 (2010)

8. Shu, H, Fan, D, Wei, J: Global stability of multi-group SEIR endemic models with distributed delays and nonlinear transmission. Nonlinear Anal., Real World Appl. 13, 1581-1592 (2012)

9. Ding, D, Ding, X: Global stability of multi-group vaccination endemic models with delays. Nonlinear Anal., Real World Appl. 12, 1991-1997 (2011)

10. Chen, H, Sun, J: Global stability of delay multigroup endemic models with group mixing and nonlinear incidence rates. Appl. Math. Comput. 218, 4391-4400 (2011)

11. Alexanderian, A, Gobbert, MK, Rister, KR, Gaff, H, Lenhart, S, Schaefer, E: An age-structured model for the spread of epidemic cholera: analysis and simulation. Nonlinear Anal., Real World Appl. 12, 3483-3498 (2011)

12. Liu, Y, Guo, S, Luo, Y: Impulsive epidemic model with differential susceptibility and stage structure. Appl. Math. Model. $36,370-378(2012)$

13. Zhang, X, Huo, H, Sun, X, Fu, Q: Impulsive epidemic model with differential susceptibility and stage structure. Appl. Math. Model. 36, 370-378 (2012)

14. Shi, X, Cui, J, Zhou, X: Stability and Hopf bifurcation analysis of an eco-epidemic model with a stage structure. Nonlinear Anal. TMA 74, 1088-1106 (2011)

15. Wu, C, Weng, P: Stability analysis of a SIS model with stage structured and distributed maturation delay. Nonlinear Anal. TMA 71, e892-e901 (2009)

16. Inaba, H: Stability analysis of a SIS model with stage structured and distributed maturation delay. Math. Biosci. 201, 15-47 (2006)

17. Feng, Z, Huang, W, Castillo-Chavez, C: Global behavior of a multi-group SIS epidemic model with age structure J. Differ. Equ. 218, 292-324 (2005)

18. Wang, W: Global behavior of an SEIR epidemic model with two delays. Appl. Math. Lett. 15, 423-428 (2002)

19. Li, MY, Smith, HL, Wang, L: Global dynamics of an SEIR epidemic model with vertical transmission. SIAM J. Appl. Math. 62, 58-69 (2001)

20. Sahu, GP, Dhar, J: Analysis of an SVEIS epidemic model with partial temporary immunity and saturation incidence rate. Appl. Math. Model. 36, 908-923 (2012)

21. Zhang, T, Teng, Z: Extinction and permanence for a pulse vaccination delayed SEIRS epidemic model. Chaos Solitons Fractals 39, 2411-2425 (2009)

22. Meng, $X$, Jiao, J, Chen, L: Two profitless delays for an SEIRS epidemic disease model with vertical transmission and pulse vaccination. Chaos Solitons Fractals 40, $2114-2125$ (2009)

23. Jiang, Y, Wei, H, Song, X, Mei, L, Su, G, Qiu, S: Global attractivity and permanence of a delayed SVEIR epidemic model with pulse vaccination and saturation incidence. Appl. Math. Comput. 213, 312-321 (2009)

24. Xu, R: Global stability of a delayed epidemic model with latent period and vaccination strategy. Appl. Math. Model. 36, 5293-5300 (2012)

25. Van den Driessche, P, Watmough, J: Reproduction numbers and sub-threshold endemic equilibria for compartmental models of disease transmission. Math. Biosci. 180, 29-48 (2002)

26. Berman, A, Plemmons, RJ: Nonnegative Matrices in Mathematical Science. Academic Press, New York (1979)

27. Freedman, HI, Tang, MX, Ruan, SG: Uniform persistence of flows near a closed positively invariant set. J. Dyn. Differ. Equ. 6, 583-600 (1994)

28. Li, MY, Graef, JR, Wang, L, Karsai, J: Global dynamics of a SEIR model with varying total population size. Math. Biosci. 160, 191-213 (1999)

29. Bhatia, NP, Szegö, GP: Dynamics Systems: Stability Theory and Applications. Springer, Berlin (1967)

30. Smith, HL, Waltman, P: The Theory of Chemostat: Dynamics of Microbial Competition. Cambridge University Press, Cambridge (1995) 